Research Paper

\title{
Effects of HMGBI Polymorphisms on the Susceptibility and Progression of Hepatocellular Carcinoma
}

\author{
Bin Wang1, Chao-Bin Yeh2,3, Ming-Yu Lein ${ }^{4,5}$, Chen-Ming Su6, Shun-Fa Yang7,8, Yu-Fan Liu ${ }^{8,9}, \varpi$, Chih-Hsin \\ Tang $4,10,11, \bowtie$ \\ 1. Department of Hepatobiliary Surgery, Affiliated Dongyang Hospital of Wenzhou Medical University, Dongyang, Zhejiang, China \\ 2. Department of Emergency Medicine, School of Medicine, Chung Shan Medical University, Taichung, Taiwan \\ 3. Department of Emergency Medicine, Chung Shan Medical University Hospital, Taichung, Taiwan \\ 4. Graduate Institute of Basic Medical Science, China Medical University, Taichung, Taiwan \\ 5. Division of Hematology and Oncology, Department of Internal Medicine, China Medical University Hospital, Taichung, Taiwan \\ 6. Department of Biomedical Sciences Laboratory, Affiliated Dongyang Hospital of Wenzhou Medical University, Dongyang, Zhejiang, China \\ 7. Institute of Medicine, Chung Shan Medical University, Taichung, Taiwan \\ 8. Department of Medical Research, Chung Shan Medical University Hospital, Taichung, Taiwan \\ 9. Department of Biomedical Sciences, Chung Shan Medical University, Taichung, Taiwan \\ 10. Department of Pharmacology, School of Medicine, China Medical University, Taichung, Taiwan \\ 11. Department of Biotechnology, College of Health Science, Asia University, Taichung, Taiwan
}

$\square$ Corresponding authors: Chih-Hsin Tang, PhD. E-mail: chtang@mail.cmu.edu.tw; Graduate Institute of Basic Medical Science, China Medical University. Yu-Fan Liu, PhD. E-mail: yfliu@csmu.edu.tw; Department of Biomedical Sciences, Chung Shan Medical University, Taichung, Taiwan.

(1) Ivyspring International Publisher. Reproduction is permitted for personal, noncommercial use, provided that the article is in whole, unmodified, and properly cited. See http://ivyspring.com/terms for terms and conditions.

Received: 2016.01.04; Accepted: 2016.03.13; Published: 2016.04.09

\begin{abstract}
Hepatocellular carcinoma (HCC) is a malignancy of liver and a leading cause of cancer mortality worldwide. Its management is compounded by biological and clinical heterogeneity. These interindividual genetic variations can modulate the effects of $\mathrm{HCC}$ treatment. High-mobility group box protein 1 (HMGBI) is a well investigated, ubiquitous nuclear protein found in eukaryotic cells that plays a multiple biological roles such as DNA stability, program cell death, immune response, and furthermore in cancer progression. In this report, we examined HMGBI single nucleotide polymorphisms (SNPs) with multiple risk factors related to HCC susceptibility and clinicopathological characteristics. Four HMGBI SNPs (rs1412125, rs2249825, rs1045411, and rs 1360485) were assessed by using a TaqMan SNPs Genotyping in 324 patients with HCC and in 695 cancer-free controls. The results showed that HMGBI SNP rs 1045411 with CT or at least one T alleles has lower risk of HCC than wild-type (CC) carriers. Moreover, HMGBI SNP rs 1412125 with TT allele has a higher risk of distant metastasis compared with patients carrying at least one $C$ allele. The present study is the first report to discuss the risk factors associated with HMGBI SNPs in HCC progression in Taiwan.
\end{abstract}

Key words: HMGB1; HCC; SNP; Susceptibility

\section{Introduction}

Worldwide, hepatocellular carcinoma (HCC) is the sixth most prevalent cancer and the third leading cause of cancer-related deaths [1]. The global incidence of HCC varies considerably, with particularly high rates in Southeast Asia and sub-Saharan Africa, and lower, but increasing rates, in North America and most of Europe [2]. In Taiwan, HCC is the second leading cause of cancer-related deaths $[3,4]$. Enormous studies have indicated that high percentage of HCC progress with chronic liver disease. The progression of HCC is a multiple process which is affected by hepatitis B virus or hepatitis C virus infection, liver fibrosis and cirrhosis, alcohol addiction and hereditary $[5,6]$.

High-mobility group box protein 1 (HMGB1) is a highly conserved, well studied, ubiquitous nuclear protein that is found in mammals [7, 8]. HMGB1 has DNA binding domains which helps stabilizing nuclear homeostasis and DNA repair [9]. HMGB1 is also expressed in cytosol and secreted into the 
extracellular space. Therefore, HMGB1 has enormous biological functions and serves as key component in enormous diseases such as inflammatory diseases and tumor [10-14].

Genetic variation plays a crucial role in an individual's susceptibility and progression to cancer. Currently, genotyping single nucleotide polymorphism (SNPs) of a population and comparing the distribution frequency of SNPs among subgroups (e.g., patients and controls) is commonly used to evaluate the risk and prognosis of a cancer [15]. Accumulating evidence suggests an association between SNPs in certain genes and HCC susceptibility. For example, specific SNPs in cathepsin $\mathrm{B}$, the enhancer of zeste 2 (EZH2) gene and plasminogen activator inhibitor contribute to the development of HCC [16-18].

HMGB1 is implicated in HCC development and progression [19]. However, the correlation between HMGB1 SNPs, HCC risk and prognosis is poorly discussed. Therefore, we investigated a case-control study of four SNPs of HMGB1 to examine the correlation between these four SNPs and HCC susceptibility and clinicopathologic characteristics.

\section{Materials and Methods}

\section{Enrollment of participants and collection of specimens}

This study consisted of 324 patients of histologically confirmed HCC from 2007 to 2012 at the Chung Shan Medical University Hospital, Taiwan. All 695 control subjects were recruited at the same hospital without previous cancer history. All the subjects in the study were Han Chinese with the same ethnicity. The patients with HCC were staged according to the Tumor size, Lymph Nodes affected, Metastases (TNM) staging system developed by the American Joint Committee on Cancer (2002) [20]. The questionnaire survey was performed with study subjects to obtain information of sociodemographic characteristics, cigarette smoking and alcohol consumption status. All clinicopathological characteristics were verified by chart review. Diagnosis of liver cirrhosis was assessed by biopsy, MRI, CT or biochemical evidence of liver parenchymal damage with endoscopic esophageal or gastric varices.

The blood samples which obtained from the controls and HCC patients were stored in EDTA tubes, centrifuged immediately and stored at $-80^{\circ} \mathrm{C}$. The Institutional Review Board of Chung Shan Medical University Hospital and informed written consent of all subjects were obtained before this study.

\section{Genomic DNA extraction}

Total genomic DNA from whole blood specimens were isolated by QIAamp DNA blood mini kits (Qiagen, Valencia, CA), following the manufacturer's instructions. DNA was dissolved in TE buffer [10 mM Tris ( $\mathrm{pH} 7.8), 1 \mathrm{mM}$ EDTA] and stored at $-20^{\circ} \mathrm{C}$ until performing Real-time quantitative PCR analysis.

\section{Real-time quantitative PCR}

Total four SNPs of HMGB1 (rs1412125, rs2249825, rs1045411, and rs1360485) were examined by using TaqMan SNPs Genotyping Assays (Applied Biosystems, Warrington, UK), according to the manufacturer's protocols. For the study, genotyping was performed in a blinded fashion without clinical data, and $10 \%$ of assays were repeated from different batches for monitoring genotyping quality. Several cases of each genotype were further examined by the DNA sequence analysis to validate results from the PCR analysis [21, 22].

\section{Statistical analysis}

Genotype distribution of each SNP was used to assess Hardy-Weinberg equilibrium (HWE) and confirmed by chi-square analysis. The distributions of demographic characteristics between control individuals and patients with HCC were examined by using Mann-Whitney U test and Fisher's exact test. The correlation between genotype frequencies, HCC cancer risk and clinicopathologic characteristics were assessed by adjusted odds ratios (AORs) with 95\% confidence intervals (CIs). Multiple logistic regression models were utilized to calculate the estimates after controlling for age, gender, alcohol consumption, and tobacco use. A $p$ value of $<0.05$ was considered statistically significant. Data were analyzed using SAS statistical software (Version 9.1, 2005; SAS Institute Inc., Cary, NC).

\section{Results}

Sociodemographic characteristics and clinical parameters for both the healthy controls and patients with HCC are shown in Table 1. HCC patients were predominantly male with a mean age of approximately 63 years. A significant association was observed between HCC development and alcohol consumption $(p<0.001)$, whereas no such significant between-group difference was found in the distribution of tobacco use $(p=0.738)$ between healthy controls and patients with HCC. To reduce possible interference of confounding variables, AORs with 95\% CIs were estimated by multiple logistic regression models after controlling for age and gender in each comparison. 
Genotype distributions and the association between HCC and healthy controls with HMGB1 polymorphisms are shown in Table 2. All selected gene markers in our control group were statistically analyzed and proved to be in the Hardy-Weinberg equilibrium $(p>0.05)$. The alleles with the highest distribution frequency at HMGB1 rs1412125, rs2249825, rs1045411, and rs1360485 in both HCC patients and controls were homozygous $\mathrm{T} / \mathrm{T}$, homozygous $\mathrm{C} / \mathrm{C}$, homozygous $\mathrm{C} / \mathrm{C}$, and homozygous $\mathrm{T} / \mathrm{T}$. Individuals carrying $\mathrm{CT}$ or $\mathrm{CT}+\mathrm{TT}$ at rs1045411 showed a 0.716-fold (95\% CI: 0.533-0.961, $p<0.05$ ) and a 0.724 -fold (95\% CI: 0.546-0.961, $p<0.05$ ) lower risk of HCC. Individuals with polymorphisms at rs1412125, rs2249825 and rs1360485 showed no reduction in HCC risk compared with wild-type individuals.

HMGB1 genotypes in HCC patients were evaluated to clarify the role of HMGB1 polymorphisms in regard to clinical TNM stage, primary tumor size, lymph node metastasis, distant metastasis, vascular invasion, Child-Pugh grade, presence of an HBV or HCV infection, and liver cirrhosis. No significant differences were observed between HMGB1 rs1412125 genotypes and clinicopathlogic status, except distant metastasis (OR: 0.309 ; 95\% CI: $0.099-0.960 ; p<0.05)$, as shown in Table 3.

AFP, AST, and ALT are common clinical pathological markers of HCC [23]. We therefore examined potential associations between the HMGB1 gene polymorphisms and levels of several serum markers of HCC. No significant associations were found between the levels of these HCC clinical pathologic markers and genotypes of any HMGB1 SNPs in HCC patients (Table 4).

Table 1. The distributions of demographical characteristics and clinical parameters in 695 controls and 324 patients with HCC.

\begin{tabular}{llll}
\hline Variable & Controls (N=695) & Patients (N=324) & $\mathrm{p}$ value \\
\hline Age (yrs) & Mean \pm S.D. & Mean \pm S.D. & \\
& $52.17 \pm 10.11$ & $62.83 \pm 11.77$ & $\mathrm{p}<0.001^{*}$ \\
Gender & $\mathrm{n}(\%)$ & $\mathrm{n}(\%)$ & \\
Male & $570(82.0 \%)$ & $233(71.9 \%)$ & \\
Female & $125(18.0 \%)$ & $91(28.1 \%)$ & $\mathrm{p}<0.001^{*}$ \\
Alcohol consumption & & & \\
No & $578(83.2 \%)$ & $201(62.0 \%)$ & \\
Yes & $117(16.8 \%)$ & $123(38.0 \%)$ & $\mathrm{p}<0.001^{*}$ \\
Tobacco consumption & & & \\
No & $402(57.8 \%)$ & $191(59.0 \%)$ & \\
Yes & $293(42.2 \%)$ & $133(41.0 \%)$ & $\mathrm{p}=0.738$ \\
Stage & & & \\
I+II & & $214(66.0 \%)$ & \\
III+IV & & $110(34.0 \%)$ & \\
Tumor T status & & & \\
$\leq \mathrm{T} 2$ & & $217(67.0 \%)$ & \\
$>$ T2 & & $107(33.0 \%)$ & \\
Lymph node status & & $313(96.6 \%)$ & \\
N0 & & $11(3.4 \%)$ & \\
N1+N2 & & \\
Metastasis & & $306(94.4 \%)$ & \\
M0 & & $18(5.6 \%)$ & \\
M1 & & $267(82.4 \%)$ & \\
vascular invasion & & $57(17.6 \%)$ & \\
No & & & \\
Yes & & & \\
Mann-Whitney U test or Fisher's exact test was used between controls and patients \\
with HCC. \\
* p value < 0.05 as statistically significant & & \\
\end{tabular}

Table 2. Distribution frequency of HMGBI genotypes in 695 controls and 324 patients with HCC.

\begin{tabular}{|c|c|c|c|c|}
\hline Variable & Controls $(\mathrm{N}=695) \mathrm{n}(\%)$ & Patients $(\mathrm{N}=324) \mathrm{n}(\%)$ & OR $(95 \% \mathrm{CI})$ & AOR (95\% CI) \\
\hline \multicolumn{5}{|l|}{ rs1412125 } \\
\hline $\mathrm{TT}$ & $374(53.8 \%)$ & $173(53.4 \%)$ & 1.00 & 1.00 \\
\hline $\mathrm{TC}$ & $275(39.6 \%)$ & $130(40.1 \%)$ & $1.022(0.776-1.346)$ & $0.833(0.583-1.190)$ \\
\hline $\mathrm{CC}$ & $46(6.6 \%)$ & $21(6.5 \%)$ & $0.987(0.571-1.705)$ & $0.889(0.456-1.732)$ \\
\hline $\mathrm{TC}+\mathrm{CC}$ & $321(46.2 \%)$ & $151(46.6 \%)$ & $1.017(0.781-1.325)$ & $0.841(0.600-1.181)$ \\
\hline \multicolumn{5}{|l|}{ rs2249825 } \\
\hline $\mathrm{CC}$ & $521(75.0 \%)$ & $235(72.5 \%)$ & 1.00 & 1.00 \\
\hline CG & $163(23.5 \%)$ & $83(25.6 \%)$ & $1.129(0.831-1.533)$ & $1.380(0.940-2.024)$ \\
\hline GG & $11(1.5 \%)$ & $6(1.9 \%)$ & $1.209(0.442-3.309)$ & $1.262(0.362-4.399)$ \\
\hline $\mathrm{CG}+\mathrm{GG}$ & $174(25.0 \%)$ & $89(27.5 \%)$ & $1.134(0.842-1.528)$ & $1.371(0.994-1.993)$ \\
\hline \multicolumn{5}{|l|}{ rs1045411 } \\
\hline $\mathrm{CC}$ & $425(61.2 \%)$ & $223(68.8 \%)$ & 1.00 & 1.00 \\
\hline $\mathrm{CT}$ & $239(34.4 \%)$ & $89(27.5 \%)$ & $\begin{array}{l}0.710(0.530-0.951)^{*} \\
p=0.022\end{array}$ & $\begin{array}{l}0.716(0.533-0.961)^{*} \\
p=0.026\end{array}$ \\
\hline TT & $31(4.4 \%)$ & $12(3.7 \%)$ & $0.738(0.372-1.465)$ & $0.794(0.398-1.584)$ \\
\hline $\mathrm{CT}+\mathrm{TT}$ & $270(38.8 \%)$ & $101(31.2 \%)$ & $\begin{array}{l}0.713(0.539-0.944)^{*} \\
p=0.018\end{array}$ & $\begin{array}{l}0.724(0.546-0.961)^{*} \\
p=0.025\end{array}$ \\
\hline \multicolumn{5}{|l|}{ rs1360485 } \\
\hline TT & $399(57.4 \%)$ & $192(59.3 \%)$ & 1.00 & 1.00 \\
\hline $\mathrm{TC}$ & $257(37.0 \%)$ & $188(36.4 \%)$ & $0.954(0.723-1.260)$ & $1.086(0.764-1.543)$ \\
\hline $\mathrm{CC}$ & $39(5.6 \%)$ & $14(4.3 \%)$ & $0.746(0.396-1.407)$ & $0.935(0.438-1.998)$ \\
\hline $\mathrm{TC}+\mathrm{CC}$ & $296(42.6 \%)$ & $132(40.7 \%)$ & $0.927(0.709-1.211)$ & $1.065(0.760-1.493)$ \\
\hline
\end{tabular}


Table 3. Odds ratio (OR) and $95 \%$ confidence interval $(\mathrm{Cl})$ of clinical status and $\mathrm{HMGBI}$ rs 1412125 genotypic frequencies in $324 \mathrm{HCC}$ patients.

\begin{tabular}{|c|c|c|c|c|}
\hline \multirow[t]{2}{*}{ Variable } & \multicolumn{3}{|c|}{ Genotypic frequencies } & \multirow[b]{2}{*}{$p$ value } \\
\hline & $\mathrm{TT}(\mathrm{N}=173)$ & $\mathrm{TC}+\mathrm{CC}(\mathrm{N}=151)$ & OR $(95 \% \mathrm{CI})$ & \\
\hline \multicolumn{5}{|l|}{ Clinical Stage } \\
\hline Stage I/II & $115(66.5 \%)$ & $99(65.6 \%)$ & 1.00 & $\mathrm{p}=0.862$ \\
\hline Stage III/IV & $58(33.5 \%)$ & $52(34.4 \%)$ & $1.041(0.657-1.651)$ & \\
\hline \multicolumn{5}{|l|}{ Tumor size } \\
\hline$\leqq \mathrm{T} 2$ & $116(67.1 \%)$ & $101(66.9 \%)$ & 1.00 & $\mathrm{p}=0.975$ \\
\hline$>\mathrm{T} 2$ & $57(32.9 \%)$ & $50(33.1 \%)$ & $1.007(0.633-1.602)$ & \\
\hline \multicolumn{5}{|c|}{ Lymph node metastasis } \\
\hline No & $167(96.5 \%)$ & $146(96.7 \%)$ & 1.00 & $\mathrm{p}=0.938$ \\
\hline Yes & $6(3.5 \%)$ & $5(3.3 \%)$ & $0.953(0.285-3.188)$ & \\
\hline \multicolumn{5}{|c|}{ Distant metastasis } \\
\hline No & $159(92.3 \%)$ & $147(97.4 \%)$ & 1.00 & $\mathrm{p}=0.033^{*}$ \\
\hline Yes & $14(8.1 \%)$ & $4(2.6 \%)$ & $0.309(0.099-0.960)$ & \\
\hline \multicolumn{5}{|c|}{ Vascular invasion } \\
\hline No & $139(80.3 \%)$ & $128(84.8 \%)$ & 1.00 & $\mathrm{p}=0.297$ \\
\hline Yes & $34(19.7 \%)$ & $23(15.2 \%)$ & $0.735(0.411-1.313)$ & \\
\hline \multicolumn{5}{|c|}{ Child-Pugh grade } \\
\hline A & $130(75.1 \%)$ & $116(76.8 \%)$ & 1.00 & $\mathrm{p}=0.725$ \\
\hline $\mathrm{B}$ or $\mathrm{C}$ & $43(24.9 \%)$ & $35(23.2 \%)$ & $0.912(0.547-1.522)$ & \\
\hline \multicolumn{5}{|l|}{ HBsAg } \\
\hline Negative & $99(57.2 \%)$ & $88(58.3 \%)$ & 1.00 & $\mathrm{p}=0.848$ \\
\hline Positive & $74(42.8 \%)$ & $63(41.7 \%)$ & $0.958(0.616-1.490)$ & \\
\hline \multicolumn{5}{|l|}{ Anti-HCV } \\
\hline Negative & $93(53.8 \%)$ & $78(51.7 \%)$ & 1.00 & $\mathrm{p}=0.705$ \\
\hline Positive & $80(46.2 \%)$ & $73(48.3 \%)$ & $1.088(0.703-1.685)$ & \\
\hline \multicolumn{5}{|c|}{ Liver cirrhosis } \\
\hline Negative & $31(17.9 \%)$ & $34(22.5 \%)$ & 1.00 & $\mathrm{p}=0.303$ \\
\hline Positive & $142(82.1 \%)$ & $117(77.5 \%)$ & $0.751(0.436-1.295)$ & \\
\hline
\end{tabular}

The ORs with analyzed by their $95 \%$ CIs were estimated by logistic regression models.

$>$ T2: multiple tumor more than $5 \mathrm{~cm}$ or tumor involving a major branch of the portal or hepatic vein(s)

* $p$ value $<0.05$ as statistically significant.

Table 4. Association of $H M G B I$ genotypic frequencies with HCC laboratory status.

\begin{tabular}{|c|c|c|c|c|}
\hline Characteristic & $\begin{array}{l}\text { a-Fetoprotein a } \\
(\mathrm{ng} / \mathrm{mL})\end{array}$ & $\begin{array}{l}\text { AST }^{a} \\
(\mathrm{IU} / \mathrm{L})\end{array}$ & $\begin{array}{l}\text { ALT }^{a} \\
(\mathrm{IU} / \mathrm{L})\end{array}$ & AST/ALT ratio a \\
\hline \multicolumn{5}{|l|}{ rs1412125 } \\
\hline $\mathrm{TT}$ & $4083.0 \pm 1321.3$ & $128.7 \pm 15.9$ & $122.6 \pm 17.8$ & $1.50 \pm 0.13$ \\
\hline $\mathrm{TC}+\mathrm{CC}$ & $2310.6 \pm 1012.3$ & $148.9 \pm 30.0$ & $109.3 \pm 18.4$ & $1.50 \pm 0.10$ \\
\hline $\mathrm{p}$ value & 0.298 & 0.540 & 0.607 & 0.987 \\
\hline \multicolumn{5}{|l|}{ rs2249825 } \\
\hline $\mathrm{CC}$ & $2412.9 \pm 751.6$ & $149.5 \pm 21.9$ & $125.6 \pm 16.9$ & $1.46 \pm 0.10$ \\
\hline $\mathrm{CG}+\mathrm{GG}$ & $5485.8 \pm 2363.3$ & $107.9 \pm 13.7$ & $92.1 \pm 13.1$ & $1.60 \pm 0.14$ \\
\hline $\mathrm{p}$ value & 0.106 & 0.257 & 0.243 & 0.456 \\
\hline \multicolumn{5}{|l|}{ rs1045411 } \\
\hline $\mathrm{CC}$ & $2953.9 \pm 900.8$ & $147.6 \pm 22.9$ & $121.1 \pm 17.4$ & $1.50 \pm 0.11$ \\
\hline $\mathrm{CT}+\mathrm{TT}$ & $3916.5 \pm 1857.3$ & $117.4 \pm 14.6$ & $106.0 \pm 14.8$ & $1.50 \pm 0.13$ \\
\hline $\mathrm{p}$ value & 0.599 & 0.390 & 0.584 & 0.973 \\
\hline \multicolumn{5}{|l|}{ rs1360485 } \\
\hline TT & $2804.9 \pm 916.4$ & $146.7 \pm 23.0$ & $129.3 \pm 19.9$ & $1.49 \pm 0.12$ \\
\hline $\mathrm{TC}+\mathrm{CC}$ & $3914.6 \pm 1604.6$ & $125.6 \pm 22.1$ & $97.6 \pm 12.0$ & $1.51 \pm 0.11$ \\
\hline $\mathrm{p}$ value & 0.521 & 0.526 & 0.224 & 0.870 \\
\hline
\end{tabular}

Mann-Whitney U test was used between two groups.

a Mean \pm S.E.

\section{Discussion}

HCC is one of the most common and lethal malignancies in the world, so preventing its occurrence and reducing its mortality rate are amongst the most important challenges faced by public healthcare. Major etiologies for HCC in Taiwan include infection with $\mathrm{HBV}$ or $\mathrm{HCV}$, alcohol consumption, a history of liver cirrhosis, and family history of HCC $[24,25]$. The data in Table 1 indicate that the ratios of tobacco smokers/nonsmokers in 
control subjects (42.2: 57.8$)$ and HCC patients (41:59) were almost equal. However, the number of subjects who had consumed alcohol among patients with HCC was higher $(38 \%)$ than that among controls $(16.8 \%)$. Increasingly, reports are providing evidence showing that alcohol consumption is a risk factor for HCC [26, 27]. Our results imply that the risk of developing HCC is higher with alcohol than it is with smoking.

Accumulating evidence has shown that progressive genomic changes can potentially alter cell phenotypes and assist preneoplastic lesions to develop into HCC. Genetic polymorphisms and somatic mutations are associated with the risk of HCC [28], while multiple gene alterations (e.g., allelic deletions, insertions, polymorphisms, mutations and methylation changes) cause genetic and molecular aberrations, which are also able to lead to the formation of HCC [29]. The genetic component is therefore widely acknowledged to be a pivotal factor for HCC risk. Thus, extensive genetic information and statistical comparisons of genetic variations between patients and healthy subjects has become an accepted and practical means of searching for genetic markers that predict the risk and pathological development of HCC.

HMGB1 plays multiple roles with inside or outside of cell, such as chromatin stabilization, DNA repair, gene transcription, program cell death regulation, and immune response. In recent studies, HMGB1 has been implicated in tumor progression such as colon, breast, oral, and lung cancer [30-32]. Previous review has indicated the role of HMGB1 in HCC progression [19]. Wang et al., also has been summarized the HMGB1 signaling pathway in HCC development [33]. In addition, Bi et al., found that HMGB1 upregulated with HCC progression [34]. However, the correlation between HMGB1 polymorphisms and HCC progression is never discussed.

In present work, four HMGB1 SNPs were assessed by SNP genotyping in 324 patients with HCC and in 695 healthy controls. The result indicated that $\mathrm{CT}$ or CT + TT at rs1045411 polymorphism is significantly correlated with reduced HCC risk. In contrast, polymorphisms at rs1412125, rs2249825 and rs1360485 showed no reduction in HCC risk compared with healthy controls. We further examined the correlation between rs1045411 polymorphism and clinicopathlogic status but showed no significant differences (Supplementary Data Table S1). It is well demonstrated that polymorphisms in the 3'-flanking region of a gene could regulate gene expression [35]. The rs1045411 polymorphism locates in the 3'-flanking region and maybe affect HMGB1 gene expression. Our data indicated that individuals with
HCC carrying at least one C allele at rs1412125 showed a lower risk of distant metastasis. This SNP is indicated to regulate binding of transcription factors for example GATA-1, GATA-2, GATA-3, and Lmo2 because its location in CCAAT box of DNA binding motif [36]. The detail functional of rs1412125 has not been discussed in present study. More experiments should been perform to elucidated the role of HMGB1 polymorphism in HCC progression.

The present study provides novel insight about the SNPs in HMGB1 on HCC susceptibility and clinicopathology. However, the limitation of this work is lacked patient's survival data. Therefore, whether HMGB1 polymorphisms link survival of HCC patients are needs further examination. In addition, larger study which contains more individuals is needed to examine the functions of HMGB1 polymorphisms in HCC progression. In conclusion, this report first provides a correlation between HMGB1 polymorphisms and HCC risk. Our investigation of HMGB1 polymorphisms may provide novel insight to develop it as helpful prognosis marker for HCC treatment.

\section{Supplementary Material}

Table S1. http:/ / www.medsci.org/v13p0304s1.pdf

\section{Acknowledgments}

This work was supported by grants from the Ministry of Science and Technology of Taiwan (NSC102-2632-B-039-001-MY3; MOST103-2628-B-039002-MY3). China Medical University Hospital (DMR-105-019). Dongyang People's Hospital (2015-YB001).

\section{Competing Interests}

All of the authors confirm that they have no financial or personal relationships with other people or organizations that could inappropriately influence this work.

\section{References}

1. Siegel R, Naishadham D, Jemal A. Cancer statistics, 2012. CA Cancer J Clin. 2012; 62: 10-29.

2. Blechacz B, Mishra L. Hepatocellular carcinoma biology. Recent Results Cancer Res. 2013; 190: 1-20.

3. Bosch FX, Ribes J, Cleries R, Diaz M. Epidemiology of hepatocellular carcinoma. Clin Liver Dis. 2005; 9: 191-211, v.

4. Wu CY, Huang HM, Cho DY. An acute bleeding metastatic spinal tumor from HCC causes an acute onset of cauda equina syndrome. Biomedicine (Taipei). 2015; 5: 18.

5. Farazi PA, DePinho RA. Hepatocellular carcinoma pathogenesis: from genes to environment. Nat Rev Cancer. 2006; 6: 674-87.

6. Forner A, Llovet JM, Bruix J. Hepatocellular carcinoma. Lancet. 2012; 379: 1245-55.

7. Mosevitsky MI, Novitskaya VA, Iogannsen MG, Zabezhinsky MA. Tissue specificity of nucleo-cytoplasmic distribution of HMG1 and HMG2 proteins and their probable functions. Eur J Biochem. 1989; 185: 303-10.

8. Bustin M, Lehn DA, Landsman D. Structural features of the HMG chromosomal proteins and their genes. Biochim Biophys Acta. 1990; 1049: 231-43. 
9. Bustin M. Regulation of DNA-dependent activities by the functional motifs of the high-mobility-group chromosomal proteins. Mol Cell Biol. 1999; 19: 5237-46.

10. Schmidt AM, Yan SD, Yan SF, Stern DM. The multiligand receptor RAGE as a progression factor amplifying immune and inflammatory responses. J Clin Invest. 2001; 108: 949-55.

11. Tang D, Kang R, Zeh HJ, 3rd, Lotze MT. High-mobility group box 1 and cancer. Biochim Biophys Acta. 2010; 1799: 131-40.

12. Sims GP, Rowe DC, Rietdijk ST, Herbst R, Coyle AJ. HMGB1 and RAGE in inflammation and cancer. Annu Rev Immunol. 2010; 28: 367-88.

13. Tang CH, Keng YT, Liu JF. HMGB-1 induces cell motility and alpha5beta1 integrin expression in human chondrosarcoma cells. Cancer Lett. 2012; 322: 98-106.

14. Hou CH, Fong YC, Tang CH. HMGB-1 induces IL-6 production in human synovial fibroblasts through c-Src, Akt and NF-kappaB pathways. Journal of cellular physiology. 2011; 226: 2006-15.

15. Shastry BS. SNP alleles in human disease and evolution. J Hum Genet. 2002; 47: 561-6.

16. Yu YL, Su KJ, Hsieh YH, Lee HL, Chen TY, Hsiao PC, et al. Effects of EZH2 polymorphisms on susceptibility to and pathological development of hepatocellular carcinoma. PLoS One. 2013; 8: e74870.

17. Chen TP, Yang SF, Lin CW, Lee HL, Tsai CM, Weng CJ. A4383C and C76G SNP in Cathepsin B is respectively associated with the high risk and tumor size of hepatocarcinoma. Tumour Biol. 2014; 35: 11193-8.

18. Weng CJ, Tsai CM, Chen YC, Hsieh YH, Lin CW, Liu YF, et al. Evaluation of the association of urokinase plasminogen activator system gene polymorphisms with susceptibility and pathological development of hepatocellular carcinoma. Ann Surg Oncol. 2010; 17: 3394-401.

19. Wang X, Xiang L, Li H, Chen P, Feng Y, Zhang J, et al. The Role of HMGB1 Signaling Pathway in the Development and Progression of Hepatocellular Carcinoma: A Review. International journal of molecular sciences. 2015; 16: 22527-40.

20. Vauthey JN, Lauwers GY, Esnaola NF, Do KA, Belghiti J, Mirza N, et al. Simplified staging for hepatocellular carcinoma. J Clin Oncol. 2002; 20: 1527-36.

21. Lin YJ, Ho TJ, Lin TH, Hsu WY, Huang SM, Liao CC, et al. P-coumaric acid regulates exon 12 splicing of the ATP7B gene by modulating hnRNP A1 protein expressions. Biomedicine (Taipei). 2015; 5:10.

22. Li TC, Li CI, Liao LN, Liu CS, Yang CW, Lin CH, et al. Associations of EDNRA and EDN1 polymorphisms with carotid intima media thickness through interactions with gender, regular exercise, and obesity in subjects in Taiwan: Taichung Community Health Study (TCHS). Biomedicine (Taipei). 2015; 5: 8.

23. Simpson HN, McGuire BM. Screening and detection of hepatocellular carcinoma. Clin Liver Dis. 2015; 19: 295-307.

24. Chen TH, Chen CJ, Yen MF, Lu SN, Sun CA, Huang GT, et al. Ultrasound screening and risk factors for death from hepatocellular carcinoma in a high risk group in Taiwan. Int J Cancer. 2002; 98: 257-61.

25. Chen CJ, Yu MW, Liaw YF. Epidemiological characteristics and risk factors of hepatocellular carcinoma. J Gastroenterol Hepatol. 1997; 12: S294-308.

26. Yang MD, Hsu CM, Chang WS, Yueh TC, Lai YL, Chuang CL, et al. Tumor Necrosis Factor-alpha Genotypes Are Associated with Hepatocellular Carcinoma Risk in Taiwanese Males, Smokers and Alcohol Drinkers. Anticancer Res. 2015; 35: 5417-23.

27. Urata Y, Yamasaki T, Saeki I, Iwai S, Kitahara M, Sawai Y, et al. Clinical characteristics and prognosis of non-B non-C hepatocellular carcinoma patients with modest alcohol consumption. Hepatol Res. 2015.

28. Thorgeirsson SS, Grisham JW. Molecular pathogenesis of human hepatocellular carcinoma. Nat Genet. 2002; 31: 339-46.

29. Buendia MA. Genetics of hepatocellular carcinoma. Semin Cancer Biol. 2000; 10: $185-200$

30. Ohmori H, Luo Y, Kuniyasu H. Non-histone nuclear factor HMGB1 as a therapeutic target in colorectal cancer. Expert Opin Ther Targets. 2011; 15: 183-93.

31. Reismann M, Wehrmann F, Schukfeh N, Kuebler JF, Ure B, Gluer S. Carbon dioxide, hypoxia and low $\mathrm{pH}$ lead to overexpression of c-myc and HMGB-1 oncogenes in neuroblastoma cells. Eur J Pediatr Surg. 2009; 19: 224-7.

32. Ahn MY, Kwon SM, Cheong HH, Park JH, Lee J, Min SK, et al. Toll-like receptor 7 agonist, imiquimod, inhibits oral squamous carcinoma cells through apoptosis and necrosis. J Oral Pathol Med. 2012; 41: 540-6.

33. Wang X, Xiang L, Li H, Chen P, Feng Y, Zhang J, et al. The Role of HMGB1 Signaling Pathway in the Development and Progression of Hepatocellular Carcinoma: A Review. Int J Mol Sci. 2015; 16: 22527-40.

34. Bi MR, Zhu LY, Yan BZ, Chen LY, Wang FX, Ma YJ, et al. Association of Upregulated HMGB1 and c-IAP2 Proteins With Hepatocellular Carcinoma Development and Progression. Hepat Mon. 2014; 14: e23552.

35. Duan ZX, Zhu PF, Dong H, Gu W, Yang C, Liu Q, et al. Functional significance of the TLR4/11367 polymorphism identified in Chinese Han population. Shock. 2007; 28: 160-4.

36. Mantovani R. A survey of 178 NF-Y binding CCAAT boxes. Nucleic Acids Res. 1998; 26: 1135-43. 